\title{
La conservación de las áreas naturales protegidas y los fenómenos PADDD (degradación, reducción y degazzettement de áreas naturales protegidas) ${ }^{1}$
}

\author{
Miguel Ángel Espichán Mariñas²
}

Universidad Científica del Sur

\section{RESUMEN}

En este artículo el autor realiza un análisis comparativo de la normativa anterior de áreas naturales protegidas y la Ley 26834, Ley de Áreas Naturales Protegidas, las categorías creadas, así como los mecanismos de protección de cada uno de ellas, con el propósito de identificar los vacíos normativos en dicha legislación que producen los llamados fenómenos PADDD, degradación (cambio de categoría), reducción o recorte del área, y el llamado degazzettement (desconocimiento oficial por parte de áreas o espacios protegidos).

\section{PALABRAS CLAVE}

Áreas naturales protegidas, espacios protegidos, análisis normativo, desregulación.

\begin{abstract}
In this article the author makes a comparative analysis of the previous rules of natural protected areas and Law 26834, Law of Natural Protected Areas, the categories created, as well as protection mechanisms for each of them, for the purpose of identify regulatory gaps in the legislation produced phenomena called PADDD, Degradation
\end{abstract}

1 Dicho fenómeno ya ha sido evidenciado en el interesante artículo de Mascia y Pailler 2010, que sirve de base para el presente texto. En ese artículo se entiende por evento o fenómeno $P A D D$, de manera general, al hecho que reduce la conservación de un espacio protegido efectuado a través de la degradación (cambio de categoría), reducción y/o recorte, así como la desregulación y desconocimiento oficial a espacios protegidos (degazzettement).

2 Abogado por la Universidad Nacional Mayor de San Marcos, con estudios de posgrado en Derecho Ambiental por la Pontificia Universidad Católica del Perú. Profesor del curso Desarrollo Sostenible e Institucionalidad Ambiental en la Universidad Científica del Sur. Miembro del Instituto de Estudios Críticos. 
(category change), Reduction or trim the area, and Degazzettement (official deregulation of Protected Areas).

\section{KEY WORDS}

Natural Protected Areas, Protected Areas, policy analysis, deregulation.

\section{Introducción}

Uno de los componentes principales dentro del análisis sobre la degradación (cambio de categoría), reducción o recorte, o desconocimiento oficial (degazzettement) de espacios protegidos (PADDD) es sin duda el componente normativo. Ello se debe a que en el Perú los espacios protegidos se crean a través de una norma legal; de ahí que hacer un análisis sobre la evolución de la normativa en áreas naturales protegidas, creemos, es un esfuerzo interesante que nos permitirá evidenciar si los sucesivos cambios al respecto pueden ser considerados como fenómenos PADDD.

A mayor abundamiento, debemos considerar que, durante la historia de la legislación sobre ANP, se han considerado diversas categorías de ANP e inclusive los enfoques y las herramientas de gestión han variado en el tiempo; de ahí que es fundamental verificar la real incidencia de los cambios y efectos normativos, a fin de determinar si pueden ser considerados como fenómenos PADDD, ya sea directamente o indirectamente.

\section{Metodología}

La metodología básicamente se basa en el análisis e interpretación de la normativa sobre ANP en el tiempo, incidiendo en la categorización de áreas, y algunos aspectos de gestión y manejo de estas.

Se usará una serie de principios de derecho ambiental y criterios de interpretación jurídica que permitan evidenciar la real dimensión del fenómeno planteado y los argumentos en cuestión orientados a la prevención de fenómenos o eventos PADDD en sede nacional.

\section{Descripción del análisis}

A través del presente trabajo se busca lo siguiente:

1. Hacer un análisis comparativo de la normativa anterior de ANP y la Ley 26834, Ley de Áreas Naturales Protegidas, las categorías creadas, así como los mecanismos de protección de cada uno de ellas.

2. Identificar los vacíos normativos en dicha legislación que pueden coadyuvar a la existencia de fenómenos PADDD, así como los criterios que permitan orientar o suplir las deficiencias normativas identificadas. 
4. Análisis comparativo e interpretación de la normativa sobre espacios protegidos en el Perú a lo largo del tiempo, usando principios de derecho ambiental y criterios de interpretación jurídica, lo que permitirá evidenciar cómo los sucesivos cambios normativos pueden haber provocado eventos PADDD

\subsection{La importancia de los antecedentes legislativos de los espacios protegidos}

Desde el punto de vista exclusivamente normativo, la regulación de espacios protegidos en nuestro país parece un tema relativamente nuevo; de ahí que un sector importante en el estudio del derecho ambiental ${ }^{3}$ parece tratar el tema de los espacios protegidos a partir de la Ley 26834, Ley de Áreas Naturales Protegidas y su Reglamento aprobado mediante Decreto Supremo 038-2001-AG.

Sin embargo, como bien se ha destacado en otros estudios ${ }^{4}$, es importante mirar la experiencia pasada, ya que la historia legal de los espacios protegidos tiene larga data. Se afirma incluso que podemos encontrar antecedentes de protección desde la época prehispánica, cuando los antiguos pobladores reservaron algunas islas del litoral para preservar las poblaciones de aves guaneras.

No obstante ello, es un denominador común citar como punto de partida el año 1941, cuando el gobierno de Manuel Prado Ugarteche ratificó la convención para la protección de la flora, fauna y bellezas escénicas naturales de los países de América, conocida como la Convención de Washington ${ }^{5}$, en la cual los países se comprometen a proteger y conservar el ambiente natural y establecer áreas protegidas, como parques nacionales, reservas nacionales, monumentos naturales y reservas regionales vírgenes ${ }^{6}$.

Sin embargo, no es sino hasta la creación del Parque Nacional de Cutervo, en $1961^{7}$, cuando se crea oficialmente el primer espacio protegido en el Perú, que es un hito en una larga y rica historia en materia de espacios protegidos, mucho antes de la Ley 26834, Ley de Áreas Naturales Protegidas.

3 Se debe entender por tal, en el sentido más amplio, al conjunto de normas jurídicas orientadas a la protección jurídica de las condiciones que hacen posible la vida. Al respecto, ver Brañes 2000: 17.

4 Al respecto, ver Universidad Nacional Agraria de La Molina 1995: 1.

5 Aprobada mediante la Resolución Suprema 983 del 11 de diciembre de 1941. El texto de la convención está disponible en: http://www.rree.gob.pe/portal/archivos.nsf/ce380461d66f6d0205 256d490080f2ae/216ea93c80efe026052576e1004faa5f?OpenDocument

6 Sobre los antecedentes, ver Solano 2005: 37 y ss.

7 Creado mediante Ley 13694 del 29 de setiembre de 1961. 
Ello implica hacer una revisión de nuestro enfoque sobre los espacios protegidos y poder entender su dinámica normativa. $Y$ es que plantear el estudio de los espacios protegidos, y en general cualquier otro tema, sin considerar los antecedentes, puede ser riesgoso. Para nuestro caso, no mirar los antecedentes puede legitimar eventos PADDD, lo que afecta la protección y conservación de dichos espacios protegidos.

A continuación, haremos un estudio cualitativo sobre la evolución de los espacios protegidos a través de la normativa nacional, a efectos de revisar basados en la data histórica los eventos PADDD que se pueden haber dado por efectos de las sucesiones y modificaciones normativas.

\subsection{La evolución histórica en materia de espacios protegidos: de la normativa forestal a la creación del Sinanpe}

A pesar de que existe una importante data normativa en materia de espacios protegidos, se debe advertir que no ha sido uniforme, fundamentalmente porque los espacios protegidos no han tenido siempre una normativa propia y han ido de la normativa forestal hacia una normativa propia. Recién con el Decreto Supremo 010-90-AG se conforma el Sistema Nacional de Áreas Naturales Protegidas por el Estado (Sinanpe), y se inicia formal y oficialmente el reconocimiento a los espacios protegidos en el Perú.

Ahora bien, si analizamos cuidadosamente, hasta 1990 ya existían distintas categorías de espacios protegidos, los que, sin embargo, como veremos a continuación, a través de esta modificación, no fueron tomados en cuenta. Ello permitirá comprender desde un punto de vista histórico cómo los cambios normativos en nuestro país han constituido y constituyen eventos PADDD (degazzette).

\subsection{El Decreto Ley 14552 y los primeros intentos}

Si bien inicialmente se creó una serie de espacios protegidos aislados, es con el Decreto Ley 14552, que se crea el Servicio Forestal y de Caza como organismo de derecho público interno, anexo al Ministerio de Agricultura, y los espacios protegidos quedan bajo el régimen normativo forestal. El decreto ley, en su artículo 4, reconoce como atribución y función del Servicio Forestal y de Caza: «d) Determinar los bosques y terrenos forestales del Estado que deben constituir reservas forestales, los bosques nacionales y los parques nacionales» (este resaltado y los siguientes son nuestros).

Asimismo, la citada norma, en su artículo 15, establece que se entiende por bosques nacionales a aquellos «establecidos con carácter definitivo, principalmente, con fines de protección o producción permanente 
de madera y productos forestales secundarios y tienen el carácter de inalienables».

Por otra parte, el artículo 16 establece que «el Poder Ejecutivo, a propuesta del Servicio Forestal y de Caza, establecerá y delimitará parques nacionales en bosques y terrenos forestales tanto del Estado como de particulares, pudiendo procederse a la expropiación en caso necesario»; y en el artículo 17 define a los parques nacionales como aquellos «establecidos con carácter definitivo para ser destinados a la protección y conservación de las bellezas escénicas naturales de la flora y la vida silvestre de importancia nacional, para ser puestos al servicio público, quedando reglamentada la explotación agropecuaria, forestal, la caza y la pesca».

\subsection{El Decreto Ley 21147 y las Unidades de Conservación}

A partir del Decreto Ley 14552, los espacios protegidos pasan a ser parte de la normativa forestal, criterio confirmado posteriormente con la dación del Decreto Ley 21147, Ley Forestal y de Fauna silvestre de 1975 en el que se agrupan los diversos espacios protegidos creados hasta esa fecha bajo el concepto de las denominadas Unidades de Conservación. El artículo 14 del citado decreto ley establece: «Considérase bajo el régimen de recurso forestal a las áreas necesarias para la protección, conservación y aprovechamiento de la fauna silvestre y las que tengan especial significación por sus valores históricos, paisajísticos y científicos».

Para tal fin, en el artículo 15 se señala: «Las unidades de conservación pueden ser: parques nacionales, reservas nacionales, santuarios nacionales y santuarios históricos. La declaración se hará mediante decreto supremo».

Al respecto, el Decreto Ley 21147 establece una serie de definiciones. Así, el artículo 16 define como parques nacionales a «las áreas destinadas a la protección con carácter de intangible, de las asociaciones naturales de la flora y fauna silvestre y de las bellezas paisajísticas que contienen».

El artículo 17 define a las reservas nacionales como las «áreas destinadas a la protección y propagación de especies de la fauna silvestre, cuya conservación sea de interés nacional. El aprovechamiento de sus productos será realizado por el Estado. Cuando las reservas nacionales deban ser establecidas necesariamente sobre tierras de uso agropecuario, el Ministerio de Agricultura podrá autorizar que el aprovechamiento de la fauna silvestre sea realizado por los conductores de dichas tierras y establecerá las limitaciones que compatibilicen el doble uso del área».

El artículo 18 define a los santuarios nacionales como las «áreas destinadas a proteger, con carácter de intangible, una especie o una comunidad 
determinada de plantas o animales, así como las formaciones naturales de interés científico o paisajístico».

El artículo 19 define a los santuarios históricos como las «áreas destinadas a proteger, con carácter de intangible, los escenarios naturales en que se desarrollaron acontecimientos gloriosos de la historia nacional».

Al amparo de dicho decreto, se establece en el artículo 20 la potestad de expropiación a favor del Estado de los predios de dominio privado que se requiera para el establecimiento de unidades de conservación.

La normativa de protección, a su vez, fue reforzada con el Decreto Ley 22175, Ley de Comunidades Nativas y de Desarrollo Agrario de la Selva y Ceja de Selva, el cual en su artículo 54 establece: «No podrán ser adjudicadas las zonas declaradas parques nacionales, reservas nacionales, santuarios nacionales e históricos, bosques nacionales y bosques de protección, así como las superficies necesarias para la explotación de recursos mineros metálicos e hidrocarburos En este último caso, podrá ser levantada la prohibición, siempre que la explotación agropecuaria no interfiera tal actividad».

\subsection{Análisis de las normas citadas}

Como podemos apreciar, es con el concepto de unidades de conservación que comienza a esbozarse un intento por tratar en forma unitaria y sistemática a los espacios protegidos, como son los parques nacionales, las reservas nacionales, los santuarios nacionales y los santuarios históricos. Se excluyó a los bosques nacionales y los bosques de protección. Los espacios de protección serían recogidos luego en el llamado Sistema Nacional de Unidades de Conservación ${ }^{8}$, sin considerar nuevamente a los bosques nacionales y los bosques de protección dentro de ese concepto.

No obstante ello, los bosques nacionales y los bosques de protección eran espacios que gozaban de protección de acuerdo con el artículo 54 del Decreto Ley 22175. El artículo 12 del Decreto Ley 21147 define a los bosques de protección como «aquellos que por sus características y ubicación sirven fundamentalmente para conservar los suelos y las aguas, con el objeto de proteger tierras agrícolas, infraestructura vial o de otra índole y centros poblados, así como para garantizar el aprovisionamiento de agua para consumo humano, agrícola e industrial. Los bosques de protección son intangibles y serán declarados por Resolución Suprema».

8 Posición que queda confirmada en el artículo 4 del Reglamento de Unidades de conservación del Decreto Ley 21147, aprobado mediante Decreto Supremo 160-77-AG, que establece que las unidades de conservación son los parques nacionales, las reservas nacionales, los santuarios nacionales y los santuarios históricos, que en conjunto conforman el Sistema Nacional de Unidades de Conservación. 


\subsection{El Decreto Supremo 010-90-AG y la creación del Sinanpe}

De acuerdo con lo indicado en el párrafo anterior, estimamos que fue la protección que la legislación reconocía a los bosques nacionales y los bosques de protección lo que motivó la dación del Decreto Supremo 01090-AG, que conforma el Sistema Nacional de Áreas Naturales Protegidas por el Estado. En el artículo 1 se establece la integración de los bosques nacionales y los bosques de protección.

Así, el artículo 1 conforma el mencionado Sistema Nacional de Áreas Naturales Protegidas por el Estado, al cual se integran el Sistema Nacional de Unidades de Conservación (Sinuc), los bosques nacionales, los bosques de protección, las reservas comunales, los cotos de caza y otras categorías de interés nacional que se establezcan en el sector agrario con fines de conservación.

Para ello, el citado decreto supremo crea el Programa Nacional Central de Sector Público Agrario denominado Programa Nacional de Parques Nacionales y otras Áreas Naturales Protegidas por el Estado, identificado también como Parques Nacionales-Perú9.

Asimismo, en el artículo 4 se establece que Parques Nacionales-Perú tiene a su cargo la gestión y administración de parques nacionales, reservas nacionales, santuarios nacionales y santuarios históricos del Sinuc, y de los bosques de protección y los bosques nacionales, así como la gestión de las reservas comunales y los cotos de caza.

En ese momento se reconoce oficialmente la categoría de área natural protegida a los bosques de protección y a los bosques nacionales, al ser integrados a este nuevo sistema erigido sobre la base del Sistema de Unidades de Conservación.

\subsection{El Decreto Supremo 011-96-AG y la exclusión de los bosques nacio- nales del Sinanpe}

No obstante la conformación del Sistema Nacional de Áreas Naturales Protegidas, con la dación del Decreto Supremo 011-96-AG, que determina zonas de protección ecológica de la región selva, ocurre uno de los más importantes fenómenos PADDD en nuestro sistema jurídico en la variante degazzette, como veremos a continuación.

9 Como organismo público descentralizado de la Dirección General Forestal y de Fauna (DGFF), con autonomía económica, técnica y administrativa en los asuntos de su competencia, que tiene como objetivo la gestión, administración, promoción y desarrollo del Sinanpe, para beneficio del pueblo peruano. Propone la normatividad relativa a sus fines y tiene iniciativa en el establecimiento de nuevas Áreas Naturales Protegidas por el Estado (ANPE). Sus acciones se realizan fundamentalmente a favor del desarrollo de las poblaciones locales. 
La citada norma surge en un contexto diferente del de la creación de áreas naturales protegidas, como uno de los reglamentos o normas complementarias a la Ley 26505 , sobre la inversión privada en el desarrollo de las actividades económicas en las tierras del territorio nacional y de las comunidades campesinas de 1995. La norma básicamente buscó promover la inversión privada en el desarrollo de las actividades económicas en las tierras del territorio nacional y de las comunidades nativas y campesinas. Para efectos de darlas en concesión, en su artículo 12 estableció que el Poder Ejecutivo determinará mediante decreto supremo las zonas de protección ecológica de la selva, en las cuales solo podrán ser materia de concesión sujetas a las normas de protección del medio ambiente.

En ese marco se promulga el Decreto Supremo 011-96-AG, cuyo artículo 1 establece que las zonas de protección ecológica «son aquellas áreas geográficas que, por sus especiales características ambientales, protegen suelos, aguas, diversidad biológica, valores escénicos, culturales, científicos y recreativos, que solo pueden ser sujetas de uso sostenible compatible con su naturaleza. Dichas zonas comprenden las siguientes áreas:

a. Las áreas naturales protegidas del Sinanpe, creado por Decreto Supremo 010-90-AG, las zonas reservadas y las áreas naturales protegidas establecidas por los gobiernos regionales, ubicadas en la región de selva, que se rigen por las normas legales de la materia.

b. Las tierras de protección en laderas, de acuerdo con lo establecido en el Reglamento de Clasificación de Tierras.

c. Las áreas de pantanos, aguajales y cochas determinadas en el mapa forestal del Perú.

d. Las áreas adyacentes a los cauces de los ríos, según la delimitación establecida por la Autoridad de Aguas».

Hasta allí todo parecería correcto. Sin embargo, en su artículo 6 excluye del Sinanpe a los bosques nacionales, por estar destinados a la producción permanente de madera, otros productos forestales y de fauna silvestre, conforme lo establecido en el artículo 10 de la Ley Forestal y de Fauna Silvestre, dada por Decreto Ley 21147.

Este artículo configura otro evento PADDD, en tanto resta una categoría reconocida a los bosques nacionales en el Decreto Supremo 010-90AG como área natural protegida integrante del Sinanpe ${ }^{10}$.

10 Uno de los ejemplos claros es el caso del Abanico del Pastaza, uno de los humedales protegidos por la convención Ramsar de gran importancia. Alcanza alrededor de 3.827.328,88 hectáreas y se habría dejado fuera de la protección del Sinanpe sin contar hasta la fecha con una mayor protección. En la década de 1940 fue creado como bosque nacional a cargo de la Dirección General Forestal del Instituto Nacional de Recursos Naturales. Sobre los datos 
Sin embargo, ello no queda allí, pues si analizamos a profundidad el artículo 6 del Decreto Supremo 011-96-AG, va contra la norma que le da origen, vale decir, la Ley 26505, la cual, en su artículo 3, establecía que para efectos de dicha ley «las áreas naturales protegidas por la Ley Forestal y de Fauna Silvestre mantienen su intangibilidad». Ello da cuenta aparentemente que una norma (Decreto Supremo 011-96-AG) que debía reglamentar una ley (Ley 26505) fue más allá de esta, pues la Ley 26505 establece que las áreas naturales protegidas (vale decir, todas las que conformaban el Sinanpe, entre ellas los bosques nacionales) en ese momento mantenían su intangibilidad. Sin embargo, el artículo 6 del Decreto Supremo 011-96-AG traspuso las barreras de lo que estaba autorizado a reglamentar.

Por otra parte, el Decreto Supremo 011-96-AG fue derogado un año después mediante el Decreto Supremo 011-97-AG, Reglamento de la Ley 26505 , referida a la inversión privada en el desarrollo de actividades económicas en tierras del territorio nacional y de las comunidades campesinas y nativas. Ese texto reproduce, en su artículo 5, lo planteado en el artículo 3 de la Ley 26505, en tanto establece que las áreas naturales protegidas por la Ley Forestal y de Fauna Silvestre mantienen su intangibilidad. No obstante, ya los bosques nacionales salieron del Sinanpe por efectos del Decreto Supremo 011-96-AG.

Como puede apreciarse, la dación del Decreto Supremo 011-96-AG y la exclusión de los bosques nacionales no fueron respetuosos de la legalidad y de por sí restaron valor a un área natural protegida como lo fueron los bosques nacionales. A todas luces, esta decisión debe ser considerada como un evento PADDD; las consecuencias muestran cómo la dinámica normativa sobre las ANP puede causar desprotección de los espacios protegidos.

\subsection{El caso particular de las zonas de reserva turística}

Otro de los casos que hemos podido encontrar en el ordenamiento jurídico peruano, de menor notoriedad que los anteriores, es el caso de las zonas de reserva turística que datan de las décadas de 1980 y 1990, básicamente sobre la base legal del Decreto Supremo 010-81-ITI/TUR-SE, el Reglamento de la Ley 24027 (Ley General de Turismo, aprobado por Decreto Supremo 039-85-ICTI-TUR), la Ley 24027 (Ley General de Turismo),

del abanico del Pastaza puede revisarse la evaluación ecológica del abanico del río Pastaza elaborado por el Centro de Datos para la Conservación de la Universidad Nacional Agraria de la Molina y la WWF, disponible en: http://www.ibcperu.org/doc/isis/5537.pdf, y la ficha informativa de los humedales de Ramsar Perú, disponible en la página del Ministerio del Ambiente: http://www.minam.gob.pe. 
y su Reglamento aprobado por Decreto Supremo 039-85-ICTI-TUR, así como la Ley 26961 (Ley para el Desarrollo de la Actividad Turística). Esta última norma establecía, en su artículo 14, que las declaraciones de zonas de reserva turística se formularán en áreas de comprobado potencial turístico que amerite protección por parte del Estado, a fin de salvaguardar el recurso de acciones que generen depredación o alteración.

Con dicha definición se hace evidente la intención de dar protección a ciertos espacios por su belleza escénica u otra cualidad para desarrollar turismo. Sin embargo, todas estas declaraciones fueron derogadas por la Resolución Ministerial 119-2011-MINCETUR-DM.

Si bien este hecho se fundamenta en que varios de los espacios declarados fueron hechos sobre espacios que fueron luego categorizados como áreas naturales protegidas, habría que verificar si todos los casos han sido absorbidos. En caso contrario, podríamos estar también ante otro fenómeno PADDD.

\section{Conclusiones. Identificación de vacíos en la legislación vigente relativa a ANP y que puede ocasionar y facilitar la aparición de eventos PADDD}

En el Perú, como en cualquier otro país, las áreas naturales protegidas representan uno de los esfuerzos globales para conservar la biodiversidad. Para ello, debido a su importancia, su creación ha sido potestad del Estado. Pese a que se reconocen numerosas iniciativas privadas, siempre se reconducen al Estado, por lo que podemos afirmar la necesaria participación estatal en la creación de áreas naturales protegidas.

Teniendo en cuenta lo antedicho, el instrumento del Estado para hacer efectiva esta preocupación y necesidad de protección ha sido encargar la gestión de las áreas y los espacios protegidos a las áreas administrativas correspondientes; de ahí es que a través de las normas jurídicas se ha propiciado la creación de espacios protegidos en nuestro país.

Hasta este punto parecería no haber novedad, aunque es precisamente la utilización de las normas jurídicas la que abre la posibilidad de los fenómenos PADD, si es que no han considerado debidamente algunos aspectos técnicos esenciales, especialmente en el caso de las modificaciones normativas.

En el Perú, la visión sobre la norma jurídica es aún una visión predominantemente estática, vale decir, una visión que se reduce a describir la norma jurídica, e identificar los supuestos de hechos regulados y las consecuencias jurídicas que aparejan dichos supuestos de hecho (por ejemplo, la creación de una reserva nacional, la identificación del espacio físico de la reserva, la categoría de protección, los usos permitidos y no permitidos), 
pero poco se ha reparado en la dinámica de las normas jurídicas, que básicamente se sustancia en las modificaciones y sucesiones normativas.

En efecto, el fenómeno de las modificaciones normativas tiene varias implicancias que no han sido tratadas y pocas veces estudiadas y consideradas por los juristas y las autoridades administrativas.

Pese a ello, existen espacios normativos en los cuales la preocupación por las modificaciones normativas y los efectos en los ciudadanos han determinado que se establezca una serie de garantías. Por ejemplo, en materia penal y laboral de la retroactividad benigna y la implícita prohibición de la normativa que no otorgue beneficios al reo o al trabajador; o, en materia procesal, la prohibición de la llamada reformatio in pejus, que prohíbe que un órgano jurisdiccional superior resuelva en perjuicio de lo ya obtenido por el apelante (es decir, puede mejorar la resolución apelada pero no empeorarla).

Como podemos ver, hay algunas garantías para el ciudadano en particular por el fenómeno de las modificaciones normativas. Sin embargo, aún son tímidos esfuerzos. El caso es que para lo concerniente a la regulación sobre espacios protegidos no hemos encontrado criterios que cautelen su intangibilidad ante el fenómeno de los cambios normativos; de ahí que la preocupación en esta parte del estudio procura hacer notar precisamente cómo este fenómeno de la variación y dinámica normativa se configura con uno de los fenómenos PADDD descritos líneas arriba.

En ese sentido, creemos que debe tenerse en claro, de acuerdo con el artículo 1 de la Ley 26834, Ley de Áreas Naturales Protegidas, que las ANP constituyen patrimonio de la nación. Su condición natural debe ser mantenida a perpetuidad, y puede permitirse el uso regulado del área y el aprovechamiento de recursos, o determinarse la restricción de los usos directos.

Ese deber es plenamente concordante con el contenido en el artículo 68 de la Constitución Política, por el cual el Estado está obligado a promover la conservación de la diversidad biológica y de las áreas naturales protegidas ${ }^{11}$.

En ese sentido, podemos afirmar que tenemos los fundamentos para establecer con claridad un principio normativo que prohíba y establezca mecanismos de tutela frente a los eventos PADDD en áreas naturales protegidas, aunque, como puede apreciarse, eso es fruto de la interpretación. Tal vez por ello es preciso y necesario establecer una regulación que explícitamente lo establezca.

11 Criterio confirmado con lo resuelto en la Sentencia del Tribunal Constitucional 033432007-PA/TC. Fundamentos 6, 7, 8, 9, 10, 11 y 12. 
Sin perjuicio de lo indicado, ya el Informe de la Defensoría del Pueblo 009-2007-DP/ASPMA.CN, denominado "Superposición de lotes de hidrocarburos con áreas naturales protegidas y reservas territoriales en la Amazonía peruana» ${ }^{12}$, desprende interesantes aportes para establecer una serie de principios, criterios y pautas que orienten la aplicación e interpretación normativa en materia de espacios protegidos.

Así, por ejemplo, debemos considerar que cualquier interpretación que se realice sobre la regulación en áreas naturales protegidas, zonas de amortiguamiento y zonas reservadas debe basarse en la obligación constitucional del Estado de promover la conservación de la diversidad biológica y de las áreas naturales protegidas, según lo establecido en el artículo 68 de la Constitución, obligación vinculante a todos los órganos de la administración estatal.

Asimismo, ya el artículo 88 del Reglamento prescribe que el Estado en su conjunto debe tener en cuenta todas aquellas medidas necesarias para que las acciones de aprovechamiento de recursos naturales ubicados en áreas naturales protegidas o sus zonas de amortiguamiento aseguren su conservación, y agrega:

88.2. En toda interpretación que se haga de la norma aplicable para el caso del aprovechamiento de los recursos naturales ubicados en las áreas naturales protegidas, la autoridad competente debe aplicar los principios precautorio, preventivo, de equidad intergeneracional y la obligación del Estado de evitar cualquier riesgo o peligro que amenace la permanencia y dinámica natural a largo plazo de los ecosistemas implicados.

Debe tenerse presente que según el artículo 4.2 de la Ley 28611, Ley General del Ambiente, se establece que el diseño, aplicación, interpretación e integración de las normas ambientales de carácter nacional, regional y local se realizan siguiendo los principios, lineamientos y normas contenidas en dicha ley y, en forma subsidiaria, en los principios generales del derecho, entre los que destacan el principio precautorio, el principio de prevención y la responsabilidad ambiental. Por ello se puede afirmar en este caso la necesidad de reconocer la existencia de un principio in dubio pro ambiente, en especial para el caso de las normas vinculadas a los espacios protegidos.

Como puede apreciarse, creemos que existen fundamentos necesarios para evitar la ocurrencia de cualquier fenómeno PADDD, sea por acción u omisión estatal. Es necesaria una regulación que clarifique el hecho y llenar estos vacíos normativos.

12 Disponible en http://www.petronoticias.com/noticias.php?op=NoticiaCompleta\&id=152 


\section{REFERENCIAS BIBLIOGRÁFICAS}

BRAÑES, Raúl (2000). Manual de derecho ambiental mexicano. México D. F.: Fondo de Cultura Económica y Fundación Mexicana para la Educación Ambiental.

MASCIA, Michael B. y PAILLER, Sharon (2010). «Protected area downgrading, downsizing and degazzettement (PADD) and its conservation implications». En http://frameweb.org/adl/en-US/6303/file/890/Mascia-Pailler\%202010. pdf. Consultado el 11 de diciembre de 2011.

SOLANO, Pedro (2005). La esperanza es verde. Áreas naturales protegidas en el Perú. Lima: Sociedad Peruana de Derecho Ambiental.

UNIVERSIDAD NACIONAL AGRARIA DE LA MOLINA (1995). Las áreas naturales protegidas del Perú. Lima: Centro de Datos para la Conservación de la Universidad Nacional Agraria de La Molina.

(2002). «Complejo de humedales del abanico del río Pastaza. Loreto, Perú». Centro de Datos para la Conservación de la Universidad Nacional Agraria de La Molina y la WWF. En http://www.ibcperu.org/doc/ isis/5537.pdf. Consultado el 11 de diciembre de 2011.

Recibido: Febrero de 2012.

Aceptado: Mayo de 2012. 